\title{
MODELLING THE CHARACTERISTICS OF CONSTRICTED-ARC PLASMA IN STRAIGHT AND REVERSE POLARITY AIR-PLASMA CUTTING*
}

\author{
M.Yu. KHARLAMOV ${ }^{2}$, I.V. KRIVTSUN ${ }^{1,2}$, V.N. KORZHIK ${ }^{1,2}$, V.I. TKACHUK ${ }^{2}$, V.E. SHEVCHENKO ${ }^{1,2}$, \\ V.K. YULYUGIN ${ }^{2}$, WU BOYI ${ }^{1}$, A.I. SITKO ${ }^{2}$ and V.E. YAROSH ${ }^{2}$ \\ ${ }^{1}$ Chinese-Ukrainian E.O. Paton Welding Institute (Guangdong General Research Institute of Industrial Technology \\ (Guangzhou Research Institute of Nonferrous Metals)) \\ 363 Changxing Road, Tianhe, 510650, Guangzhou, China. E-mail: vnkorzhyk@gmail.com \\ ${ }^{2}$ E.O. Paton Electric Welding Institute, NASU \\ 11 Bozhenko Str., 03680, Kiev, Ukraine. E-mail: office@paton.kiev.ua
}

\begin{abstract}
The paper deals with the issues of mathematical modelling of turbulent flow of electric arc plasma in straight and reverse polarity plasma cutting. It is noted that the main complexity of modelling reverse polarity cutting arises at description of plasma flow in near-electrode regions. In view of that, it is proposed to describe arc electromagnetic characteristics in the cut cavity proceeding from the known experimental data. Detailed comparative analysis of the influence of plasmatron operating modes and arc polarity on arc discharge electric characteristics, thermal and gas-dynamic characteristics of the plasma flow is performed on the base of numerical modelling. 17 Ref., 1 Table, 5 Figures.
\end{abstract}

Ke $\boldsymbol{y} \boldsymbol{w} \boldsymbol{o r d} \boldsymbol{s}:$ plasma cutting, straight and reverse polarity, electric arc plasma, turbulent flow, mathematical modelling, near-electrode regions, arc discharge electric characteristics

Plasma cutting is currently widely accepted in industry, primarily owing to high productivity and accuracy of the cutting process, as well as other factors, providing competitive advantages over other thermal cutting processes [1, 2]. So, for instance, laser cutting is inferior to plasma cutting, because of the high cost, as well as significant limitation on thickness of cut blanks (approximately $4 \mathrm{~mm}$ for metals), and oxygen cutting is inferior to plasma as to cut quality, cutting process effectiveness being largely determined by oxygen purity. Here, wider application of plasma cutting in practice is restrained by a number of limitations, inherent to this technology, among which we should, first of all, note the thickness of blanks being cut, limited by approximately $70 \mathrm{~mm}$ for steels [1]. Therefore, the priority tasks set for specialists involved in development of plasma cutting technologies, are increasing maximum cut thickness, alongside increase of cutting speed and blanks cutting-out accuracy, reduction of specific energy consumption, etc. These tasks can be solved by increasing the heat input in the cut zone, as well as providing more uniform heat distribution across the cut thickness, alongside creation of favorable gas-dynamic conditions for molten material blowing out.

Technological potential for equipment development for straight polarity (SP) plasma cutting, widely applied in practice, has practically been exhausted that is related to physics of the processes occurring here. In this connection, plasma cutting with application of reverse polarity (RP) arc, is an urgent direction of this technology development, fully meeting the modern industry requirements [1-3].

However, despite the fact that RP plasma cutting has been developed since 1970s, the process is still insufficiently studied, that does not allow designing reliable high-productivity cutting plasmatrons. Now, development of plasma cutting processes, alongside creation of the respective equipment (plasmatrons), is closely connected with deep and comprehensive study of physical processes running both in such plasmatrons, and in processed material. In particular, gas-dynamic, thermal and electric characteristics of arc plasma, generated by such plasmatrons, have a strong influence on productivity, stability, as well as resulting quality characteristics of plasma-arc processes of material processing. Here, in view of the complexity of these processes, mathemati-

\footnotetext{
* The work has been performed with financial support under Foreign Expert Program in PRC No.WQ20124400119, R\&D Project of Innovation Group of Guangdong Province No.201101C0104901263 and International Project of The Ministry of Science and Technology of PRC No.2013DFR70160. 
cal modelling methods are extremely important in performance of research aimed at improvement of plasmatron designs and selection of their optimum operation modes.

Development of mathematical model of arc plasma, generated by cutting plasmatrons in their operation both at straight and reverse polarity, as well as its application for conducting numerical experiments, were exactly the objective of this work.

There is a sufficient number of publications, devoted to theoretical studies of gas-dynamic, thermal and electromagnetic processes in plasma, generated by plasmatrons operating at straight polarity (see, for instance, [4-8]). Adequate mathematical models of plasmatrons with both transferred and non-transferred arc have been developed, allowing for a multitude of factors, including geometrical parameters of plasmatron working channel, turbulent nature of plasma flow, non-uniformity of electromagnetic field [9], etc.

These models are based on a system of NavierStokes magnetogasdynamic (MGD) equations [4-6], closed by additional equations, or relationships, describing the used turbulence model, magnetic component of pressure, etc.

A simplified system of MGD equations in boundary layer approximation can be used in most of the cases, which is derived from the condition of smallness of axial gradients of temperature and velocity of plasma, compared to radial ones [10]. A simplified model is used here to describe the arc electromagnetic characteristics, which is based on the assumption of smallness of radial component of electric current density, compared to axial component $\left(j_{r}<j_{z}\right)$. In the case of transferred-arc plasmatrons, in which part of the arc is running in open space, Maxwell equation [9] is applied for a more correct description of arc electromagnetic characteristics, for the solution of which boundary conditions are distributions of arc electromagnetic charac- teristics in near-electrode regions, which are assigned proceeding from experimental data. The best studied here are the processes on refractory (tungsten) cathode and in the near-cathode region of SP arc, running in different inert gases.

On the other hand, there are no models, describing the characteristics of electric arc plasma, generated by plasmatrons with RP arcs. In particular, during plasma cutting, RP electric arc continuously changes its length, while interacting with the gas flow, electromagnetic fields, walls of the electrode, nozzle channel and cut cavity, that makes the running processes complex for mathematical description. In this connection, at construction of mathematical model of cutting plasmatron, it appears rational to apply NavierStokes MGD equations together with the data of measurement of integral and distributed characteristics of arc plasma, generated by the plasmatrons operating at reverse polarity. Data of such measurements are given, for instance, in [2], and, in fact, allow selection of distributions of plasma parameters in near-electrode regions, required for further calculations. Application of these principles was exactly the basis for construction of a mathematical model of cutting plasmatrons with the arc of both straight and reverse polarity.

Problem definition. To construct a mathematical model of plasma arc at straight and reverse polarity air-plasma cutting, let us consider plasmatron schematic, given in Figure 1. Let us assume that constricted transferred arc runs between hollow electrode (anode or cathode, depending on arc polarity) and metal being cut, closing in the cut cavity. The arc runs both in the inner regions - plasmatron channel and cut cavity, and in the open section between plasmatron nozzle edge and surface of item being cut. Hollow electrode with inner radius $R_{p}$ is located at distance $Z_{1}$ from plasmatron outlet nozzle edge with the region of arc binding at $z=Z_{p}$. Plasma gas with flow rate $G$ is fed swirled through an-

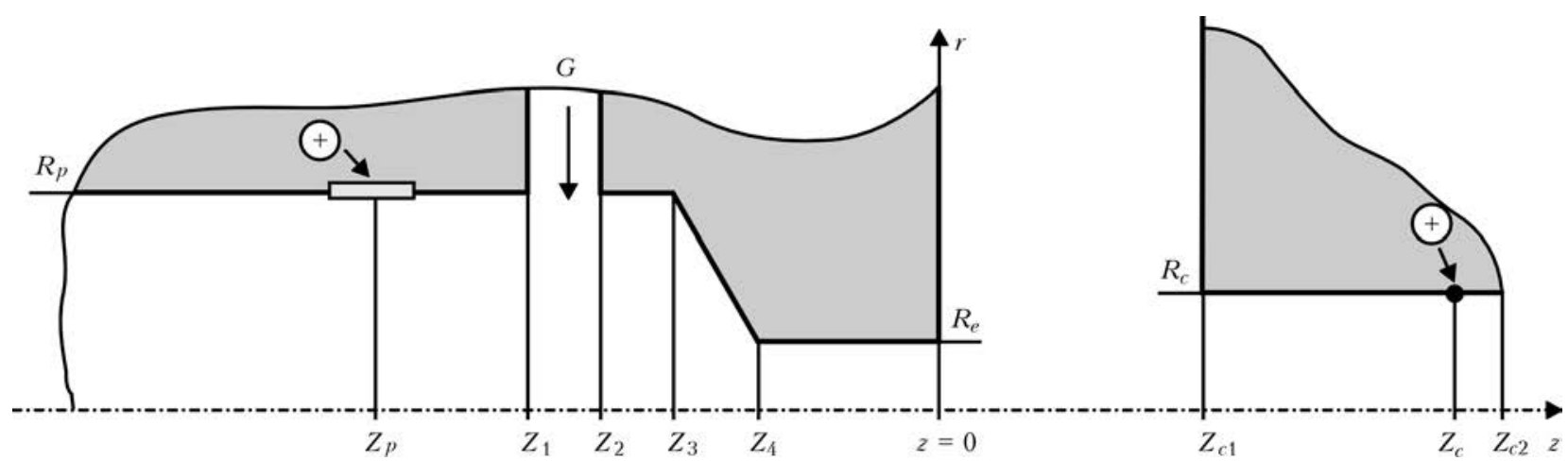

Figure 1. Calculation schematic of cutting plasmatrons and cut item in straight and reverse polarity cutting (for designations see the text) 
nular channel $Z_{1} \leq z \leq Z_{2}$ at $r=R_{p}$. Then plasma gas is heated and accelerated by the electric arc, and passing through convergent tube $\left(Z_{3} \leq \mathrm{z} \leq\right.$ $\leq Z_{4}$ ) and outlet nozzle of radius $r=R_{e}$ and length $Z_{4}$, it flows out into the environment. The arc expanding in the external area, then goes into the cut cavity, becoming constricted by its walls. Location of the arc basic spot in cut cavity $(z=$ $=Z_{c}$ ) can change within the thickness of item being cut, i.e. in region $Z_{c 1} \leq z \leq Z_{c 2}$.

We will consider the simplest variant of a stationary discharge in axially symmetric plasmatrons, operating in plasma gas turbulent flow mode, when the influence of electromagnetic factors on the nature of turbulence can be ignored, i.e. it can be considered purely gas-dynamic. The latter means that pulsations of current density, magnetic field intensity, as well as electric conductivity coefficient can be neglected, considering these values as averaged.

We will take the following assumptions for mathematical description of arc plasma flow in cutting plasmatron:

- considered plasma system has cylindrical symmetry, and the processes running in it are assumed to be stationary;

- disturbances, introduced into the flow, when bypassing the ledges in channel expansion planes, do not have any essential influence on the jet thermal and gas-dynamic characteristics;

- plasma is in the state of local thermodynamic equilibrium, plasma natural radiation is volumetric;

- main mechanism of plasma heating is Joulean heat evolution (work of pressure forces and viscous dissipation can be neglected), and energy transfer in the plasma flow occurs due to heat conduction and convection (natural convection is disregarded);

- plasma flow is viscous, subsonic, flow mode is turbulent;

- external magnetic fields are absent.

Tangential feeding of plasma gas into the plasmatron working channel and its relatively high flow rate should be also taken into account.

Basic equations. Allowing for the assumptions made, gas dynamic and thermal characteristics of plasma flow generated by cutting plasmatron can be described by the following system of MGD equations (with gas swirling) in the approximation of turbulent boundary layer for time-averaged values of plasma temperature and velocity $[5,6,10]$ :

$$
\frac{\partial}{\partial z}(\rho u)+\frac{1}{r} \frac{\partial}{\partial r}(r \rho \bar{v})=0 ;
$$

$$
\begin{gathered}
\rho\left(u \frac{\partial u}{\partial z}+\bar{v} \frac{\partial u}{\partial r}\right)=\frac{1}{r} \frac{\partial}{\partial r}\left(r \bar{\eta} \frac{\partial u}{\partial r}\right)-\frac{\partial}{\partial z}\left(p+\mu_{0} \frac{H_{\varphi}^{2}}{2}\right) ; \\
\rho\left(u \frac{\partial w r}{\partial z}+\bar{v} \frac{\partial w r}{\partial r}\right)=\frac{1}{r} \frac{\partial}{\partial r}\left(r \bar{\eta}\left(\frac{\partial r}{\partial r}-2 w\right)\right) ; \\
\rho C_{p}\left(u \frac{\partial T}{\partial z}+\bar{v} \frac{\partial T}{\partial r}\right)=\frac{1}{r} \frac{\partial}{\partial r}\left(r \bar{\chi} \frac{\partial T}{\partial r}\right)+\frac{j^{2}}{\sigma}-\psi .
\end{gathered}
$$

Here, $T$ is the averaged plasma temperature; $\bar{v}=$ $=\left(\rho v+\rho^{\prime} v^{\prime}\right) / \rho$, where $v$ is the averaged radial velocity; $\rho$ is the averaged plasma density; $\rho^{\prime}$ and $v^{\prime}$ are the pulsations of density and radial velocity; $u$ is the averaged axial plasma velocity; $w$ is the averaged azimuthal rotational speed; $p$ is the pressure; $C_{p}$ is the specific heat capacity of plasma at constant pressure; $\sigma$ is the plasma electric conductivity; $j$ is the vector of electric current density; $\psi$ is the volume density of natural radiation power; $\bar{\eta}$ and $\bar{\chi}$ are the total coefficients of dynamic viscosity and heat conductivity of plasma, which are a sum of molecular and turbulent viscosity and heat conductivity, respectively; $\mu_{0}$ is the universal magnetic constant;

$$
H_{\varphi}=\frac{1}{r} E_{z} \int_{0}^{r} \sigma r d r
$$

is the azimuthal component of arc current magnetic field, where $E_{z}$ is the axial component of electric field intensity.

Within the used boundary layer approximation, description of distributed electromagnetic characteristics of the arc is performed under the condition that $j_{r}<<j_{z}$. In this case, addendum $j^{2} / \sigma$ in equation (4), describing energy evolution in the plasma due to electric current flowing, becomes $j_{z}^{2} / \sigma$. Here, the axial component of arc electric field intensity is practically constant across channel cross-section [6], and is found from the condition of total current preservation:

$$
I=2 \pi E_{z} \int_{0}^{R_{\sigma}(z)} \sigma r d r,
$$

where $R_{\sigma}(z)$ is the radius of current-conducting region.

Considering that plasma conductivity is practically zero outside this region, calculated region radius can be used as upper limit of integration in formula $(6)$, i.e. in the channel $(z<0)$ it can be assumed equal to channel radius $R_{\sigma}(z)=$ $=R_{p}(z)$, where $R_{p}(z)=R_{p}$ at $z<Z_{2}, R_{p}(z)=$ $=R_{e}$ at $Z_{4} \leq z<0$, and $R_{p}(z)=R_{e}+((z-$ $\left.\left.Z_{4}\right) /\left(Z_{3}-Z_{4}\right)\right)\left(R_{p}-R_{e}\right)$ at $Z_{3} \leq z<Z_{4}$; in open 
region $\left(0 \leq z \leq Z_{c 1}\right) R_{\sigma}(z)-R_{\delta}(z)=\mathrm{R}$, and in cut cavity $\left(Z_{c 1}<z \leq Z_{c 2}\right)-R_{\sigma}(z)=R_{c}$ (see Figure 1).

Pressure distribution within the plasma-forming channel $(z \leq 0)$ is determined allowing for magnetic component of pressure and gas swirling:

$$
\begin{aligned}
p=p_{\text {ext }}-\int_{z}^{0} \frac{d p_{c}}{d z} d z+\mu_{0} E_{z} \int_{r}^{R_{p}(z)} \sigma H_{\varphi} d r- \\
\quad-\int_{r}^{R_{p}(z)} \frac{w^{2}}{r} d r,
\end{aligned}
$$

where $p_{\text {ext }}$ is the ambient pressure.

Gas-static pressure gradient $d p_{c} / d_{z}$ in boundary layer approximation is also constant across the channel section [10] and is determined from the conditions of preservation of total mass flow of plasma gas:

$$
\rho_{0} G=2 \pi \int_{0}^{R_{p}(z)} \rho u r d r
$$

where $\rho_{0}$ is the gas mass density under normal conditions; $G$ is the volume flow rate of gas. In the open discharge region, as well as in the cut cavity $(z>0)$ pressure is given by the following expression:

$$
p=p_{\text {ext }}+\mu_{0} E_{z} \int_{r}^{R_{c}(z)} \sigma H_{\varphi} d r-\int_{r}^{R_{c}(z)} \rho \frac{w^{2}}{r} d r .
$$

System of equations (1)-(9) is complemented by the following relationships:

$$
\begin{gathered}
\rho=\rho(T, p) ; \quad C_{p}=C_{p}(T, p) ; \quad \chi=\chi(T, p) ; \\
\eta=\eta(T, p) ; \quad \sigma=\sigma(T, p) ; \quad \psi=\psi(T, p),
\end{gathered}
$$

determining the dependencies of thermodynamic characteristics, molecular transfer coefficients and optical properties of plasma on temperature and pressure. Detailed tables of the above values for the used plasma gases are given, for instance, in $[6,11]$.

In the section of currentless (inertia) plasma flow, which under the described conditions can occur in the cut cavity beyond the arc binding region $\left(z>Z_{c}\right)$, the same system of gas-dynamic equations can be used, assuming $E_{z}=H_{\varphi}=0$.

Turbulence modelling. Coefficients of dynamic viscosity and heat conductivity of plasma used in the above equations have the following form:

$$
\bar{\eta}=\eta+\eta_{t} ; \quad \bar{\chi}=\chi+\chi_{t},
$$

where $\eta, \chi$ are the coefficients of molecular viscosity, determined according to $(9) ; \eta_{t}, \chi_{t}$ are the coefficients of turbulent viscosity and heat conductivity.

Turbulent transfer coefficients were determined using $k-\varepsilon$ model [12], in which coefficients of turbulent viscosity and heat conductivity are given by the following expressions:

$$
\begin{gathered}
\eta_{t}=\frac{C_{\mu} \rho(\bar{k})^{2}}{\varepsilon} ; \\
\chi_{t}=\eta_{t} \frac{C_{p}}{\operatorname{Pr}_{t}}
\end{gathered}
$$

where $\bar{k}$ is the kinetic turbulence energy; $\varepsilon$ is the turbulence dissipation rate; $C_{\mu}=0.09$ is the empirical constant; $\operatorname{Pr}_{t}$ is the turbulent Prandtl number, which is selected according to recommendations of [13], or is taken equal to a unity [6]. Equation of turbulent viscosity is closed by equations of transfer for kinetic energy of turbulence and dissipation rate:

$$
\begin{gathered}
\rho\left(u \frac{\partial \bar{k}}{\partial z}+\bar{v} \frac{\partial \bar{k}}{\partial r}\right)= \\
=\frac{1}{r} \frac{\partial}{\partial r}\left[r\left(\eta+\frac{\eta_{t}}{\operatorname{Pr}_{k}}\right) \frac{\partial \bar{k}}{\partial r}\right]+G-\rho \varepsilon \\
\rho\left(u \frac{\partial \bar{\varepsilon}}{\partial z}+\bar{v} \frac{\partial \bar{\varepsilon}}{\partial r}\right)= \\
=\frac{1}{r} \frac{\partial}{\partial r}\left[r\left(\eta+\frac{\eta_{t}}{\operatorname{Pr}_{\varepsilon}}\right) \frac{\partial \bar{\varepsilon}}{\partial r}\right]+C_{1} G \frac{\varepsilon}{\bar{k}}-C_{2} \rho \frac{\varepsilon^{2}}{\bar{k}} .
\end{gathered}
$$

Here, $G=\eta_{t}\left(\frac{\partial u}{\partial r}\right)^{2}$ is the source term, and $C_{1}$, $C_{2}, \operatorname{Pr}_{k} \operatorname{Pr}_{\varepsilon}$ are the constants of $k-\varepsilon$ model of turbulence, equal to $1.44,1.92,1.3$ and 1.0 , respectively.

Boundary conditions. To close the described system of equations, it is necessary to assign the boundary and initial (input) conditions, corresponding to the considered conditions of plasma cutting (see Figure 1).

The following conditions were assumed to be valid on the system axis of symmetry $(r=0)$ :

$$
\frac{\partial T}{\partial r}=0 ; \frac{\partial u}{\partial r}=0 ; \bar{v}=0 ; \frac{\partial \bar{k}}{\partial r}=0 ; \frac{\partial \varepsilon}{\partial r}=0 .
$$

On plasmatron walls, i.e. at $z \leq 0$ and $r=$ $=R_{p}(z)$, «sticking» condition is set and cooled wall temperature $T_{w}$ is assigned, i.e.

$$
u=\bar{v}=w=0 ; \quad T=T_{w} .
$$

To assign $\bar{k}$ and $\varepsilon$ values near the channel wall, it is necessary to apply the near-wall function $[12,14]$, determining the above values as follows: 


$$
\bar{k}=\frac{u_{*}^{2}}{\sqrt{C_{\mu}}}, \quad \varepsilon=\frac{u_{*}^{3}}{k_{0}\left(R_{c}-r\right)},
$$

where $k_{0}=0.41$, and $u_{*}$ is the solution of transcendental equation (logarithmic wall law):

$$
\frac{u}{u_{*}}=\frac{1}{k_{0}} \ln \left[\frac{\Lambda \rho u_{*}\left(R_{c}-r\right)}{\eta}\right] \text {, }
$$

where $\Lambda=9.0$ is the wall roughness parameter.

Expressions (17), (18) are used for correct allowance for viscous underlayer at determination of $k$ and $\varepsilon$ in near-wall region, i.e. at $y^{+}=$ $=\rho\left(R_{c}-r\right) u_{*} / \eta<f^{+}$, where $f^{+}$is selected in the range of 20-100 [14]. Equations (13), (14) of fully developed turbulent flow are used to describe the flow inner region $\left(y^{+} \geq f^{+}\right)$.

Conditions of smooth coupling with the environment are selected on the external boundary of calculated region (open section):

$$
T=T_{\text {ext }} ; \quad u=\bar{v}=w=0 .
$$

Considering the complexity of mathematical description of the region of plasma flow near the arc basic region in plasmatron discharge chamber, initial conditions were set in $z_{0}=Z_{1}$ section:

$$
\begin{gathered}
\bar{v}\left(r, z_{0}\right)=0 ; \quad u\left(r, z_{0}\right)=u_{0}(r) ; \\
w\left(r, z_{0}\right)=w_{0}(r) ; \quad T\left(r, z_{0}\right)=T_{0}(r) .
\end{gathered}
$$

Used as initial conditions $u_{0}(r), w_{0}(r), T_{0}(r)$ was the solution for arc in plasmatron channel in one-dimensional approximation (at $\bar{v}=0$ and $\left|\frac{\partial \psi}{\partial z}\right|=0$, where $\left.\psi=\{T, u, \bar{v}, r w\}\right)$ :

$$
\begin{gathered}
\frac{1}{r} \frac{\partial}{\partial r}\left(\bar{\eta} \frac{\partial u}{\partial r}\right)-\frac{\partial p}{\partial z}=0 \\
\frac{1}{r} \frac{\partial}{\partial r}\left(r \bar{\gamma} \frac{\partial T}{\partial r}\right)-\frac{j^{2}}{\sigma}-\psi=0
\end{gathered}
$$

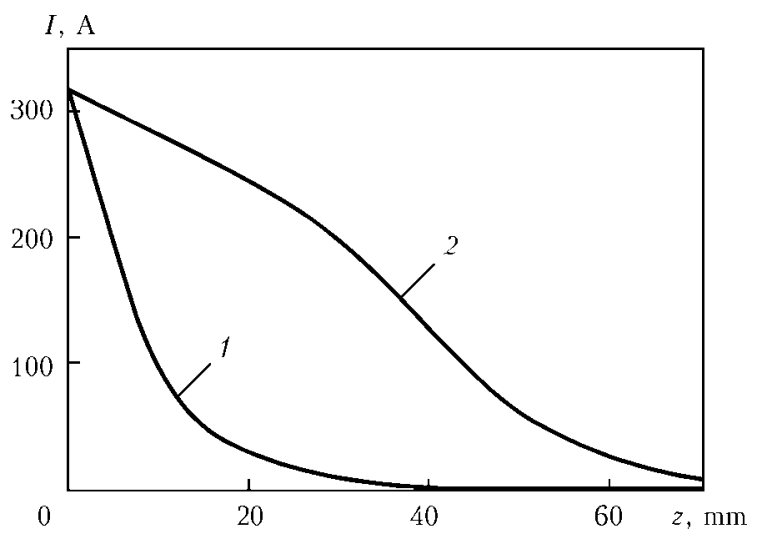

Figure 2. Change of total current of plasma arc along the cut cavity at straight (1) and reverse (2) polarity cutting at $I=315 \mathrm{~A}, G=3.4 \mathrm{~m}^{3} / \mathrm{h}, Z_{c 2}-Z_{c 1}=70 \mathrm{~mm}, R_{c}=$ $=5.5 \mathrm{~mm}$ complemented by empirical distribution of azimuthal rotational speed [6]

$$
w(r)=A\left(1-e^{-k r^{2}}\right) / r,
$$

where $A$ and $k$ constants are related to maximum speed and its coordinate $r_{m}$ :

$$
A=1.4 r_{m} \omega_{m} ; \quad k=1.25 / r_{m}^{2} ; \quad r_{m}=R / \sqrt{3} .
$$

Equations (21)-(23) were closed by expressions for arc current (6), plasma gas flow rate (8), as well as boundary conditions on the axis of symmetry (15) and walls of plasmatron channel (16). Solution of equation (21)-(23) was determined for assigned arc current $I$ and plasma gas flow rate $G_{0}=\varepsilon G$, where $\varepsilon$ was taken equal to 0.05 .

Volumetric flow rate of plasma gas through plasmatron working channel was determined allowing for vortex feeding of plasma gas via annular channel $Z_{1} \leq z \leq Z_{2}$ :

$$
G=\left\{\begin{array}{l}
0, z<Z_{1}, \\
\frac{z-Z_{1}}{Z_{2}-Z_{1}} G_{1}, Z_{1}<z<Z_{2} \\
G_{1}, Z_{2}<z<0,
\end{array}\right\}
$$

where $G_{1}$ is the volume fraction of plasma gas fed into plasmatron working channel.

And, finally, in cut cavity $\left(Z_{c 1} \leq z \leq Z_{c 2}\right)$ conditions (15), (16) were set, and arc current distribution along the cut cavity was assigned:

$$
I_{c}=I_{c}(z),
$$

which was determined proceeding from experimental data.

Current distribution along the cut cavity. To assign distribution of arc current of straight and reverse polarity along the cut cavity during plasmatron operation, experimental data [2] were used, which were the basis for construction of interpolation polynomial. Here, considering that the results of [2] were obtained for working current of $90 \mathrm{~A}$, current values in the cut cavity were determined in proportion to cutting plasmatron working current $I$, used in calculations. Figure 2 gives current distributions in the cut cavity, plotted for arc current of $315 \mathrm{~A}$.

Computer modelling results. Defined problem was solved numerically, by the method of finite differences $[15,16]$. Main difference scheme for integration of systems of equations of the type of boundary layer equation was used [17]. Second-order differential equations (2), (3), (13), (14) were approximated by implicit two-layer six-point difference scheme, and firstorder equation (1) - by explicit four-point 

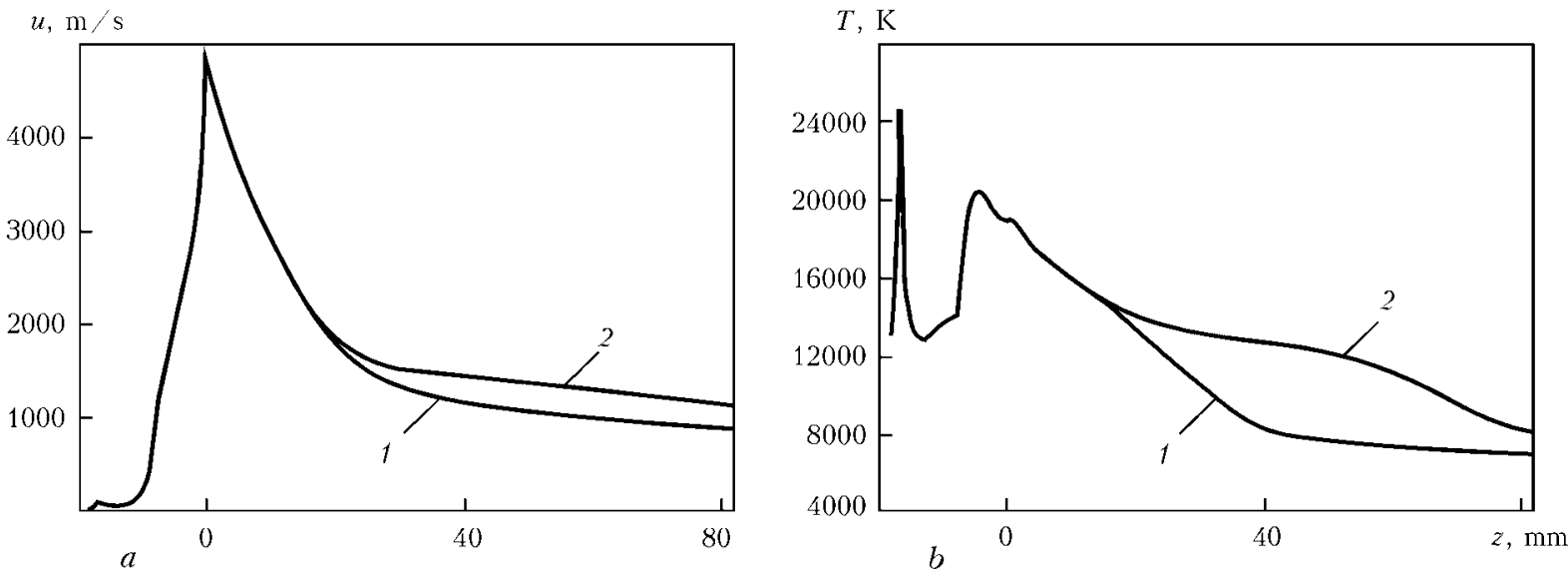

Figure 3. Distribution of axial values of plasma velocity $(a)$ and temperature $(b)$ along the axis of constricted arc generated by cutting plasmatron at its operation at straight (1) and reverse (2) polarity

scheme. Derived algebraic system of difference equations was solved by sweep method using layered iterations over $z$, with which global iterations over pressure were used to obtain a solution in cutting plasmatron channel.

Proceeding from the developed physicomathematical model, corresponding to computational scheme and software for is computer realization, numerical analysis has been performed of the characteristics of turbulent flow of arc plasma generated by the cutting plasmatron at different modes of its operation, both at straight and at reverse polarity.

During calculations, geometrical parameters of cutting plasmatron (internal dimensions of hollow anode, swirler, outlet nozzle) were assigned as follows (see Figure 1): $R_{e}=2 \mathrm{~mm}$, $Z_{4}=-8 \mathrm{~mm}, Z_{3}=-13 \mathrm{~mm}, Z_{2}=-17 \mathrm{~mm}, Z_{1}=$ $=-18.5 \mathrm{~mm}, Z_{p}=-58.5 \mathrm{~mm}, R_{p}=7 \mathrm{~mm}$. Distance to item being cut $Z_{c 1}$ was assumed to be equal to $12 \mathrm{~mm}$, thickness of cut item $Z_{c 2}-Z_{c 1}=$ $=70 \mathrm{~mm}$, cut cavity diameter $-11 \mathrm{~mm}$. The following parameters were used as the main parameters of cutting plasmatron operation: arc current $I=315$ A, plasma gas (air) flow rate $G=$ $=3.4 \mathrm{~m}^{3} / \mathrm{h}$. Ambient pressure was atmospheric.

Let us, first of all, consider the results of calculation of distributed characteristics of plasma flow, generated by cutting plasmatron at its operation at straight and reverse polarity. Calculated distributions of the values of plasma temperature and velocity along the plasma jet are given in Figure 3.

Proceeding from the presented results, plasma behaviour in the considered plasmatron, as well as in the open region and in cut cavity, can be presented as follows. Cold plasma gas entering the plasmatron via annular channel $Z_{2} \leq z \leq Z_{3}$ is gradually drawn into the flow. On the other hand, plasma flow core starts forming gradually in this flow section. However, annular near-wall flow of cold gas prevents expansion of heated plasma regions, resulting in formation of quite narrow current-conducting region, in which plasma temperature reaches the values of 15$24 \mathrm{kK}$ at quite low flow velocities.

Further plasma flow in plasmatron working channel is associated with expansion of flow core in the region $Z_{2} \leq z \leq Z_{3}$ and plasma temperature drop on the axis. Then, passing into the convergent tube $Z_{3} \leq z \leq Z_{4}$, the plasma flow is compressed, plasma velocity and temperature rising significantly. By the moment of entering plasmatron outlet nozzle $Z_{4} \leq z \leq 0$, the plasma flow practically completely fills the channel section. Being squeezed by relatively narrow walls of plasmatron outlet nozzle, the flow is intensively accelerated and heated by the electric arc, reaching the values of plasma velocity and temperature of the order of $5000 \mathrm{~m} / \mathrm{s}$ and $20 \mathrm{kK}$, respectively, on plasmatron nozzle edge.

Then plasma arc flows into open region $0 \leq$ $\leq \mathrm{z} \leq Z_{c 1}$, in which the plasma flow is somewhat expanded, that leads to lowering of the values of plasma velocity and temperature in the open region. Here, plasma in the flow is still exposed to electromagnetic forces, being in the region of electric arc impact. Then, penetrating into the cut area, the plasma arc is squeezed by its walls.

Differences in plasma flow parameters at straight and reverse polarity cutting are observed, primarily, in the cut cavity, that is associated with the nature of current distribution along the cut length in different arcing modes.

At SP cutting, plasma velocity and temperature decrease quite intensively, when going deeper into the cut cavity. In this case, the largest heat flow will come to subsurface regions of the item being cut. Now, at RP cutting distribution of plasma velocity and temperature along the cut cavity is of a more uniform nature; therefore intensive heat evolution in the arc occurs over the 
SCIENTIFIC AND TECHNICAL

Arc voltage in plasma cutting at straight and reverse polarity

\begin{tabular}{|c|c|c|c|c|c|c|}
\hline \multirow{2}{*}{ Polarity } & \multirow{2}{*}{$U_{\mathrm{a}}, \mathrm{V}$ (experiment) } & \multirow{2}{*}{$U_{\mathrm{a}}, \mathrm{V}$ (calculation) } & \multicolumn{4}{|c|}{$U, \mathrm{~V}$, at } \\
\hline & & & $Z_{p}<z<Z_{1}$ & $Z_{1}-0$ & $0-Z_{c 1}$ & $Z_{c 1}-Z_{c 2}$ \\
\hline Straight & 320 & 318 & 33 & \multirow[t]{2}{*}{173} & \multirow[t]{2}{*}{56} & 46 \\
\hline Reverse & 340 & 375 & 8 & & & 127 \\
\hline
\end{tabular}
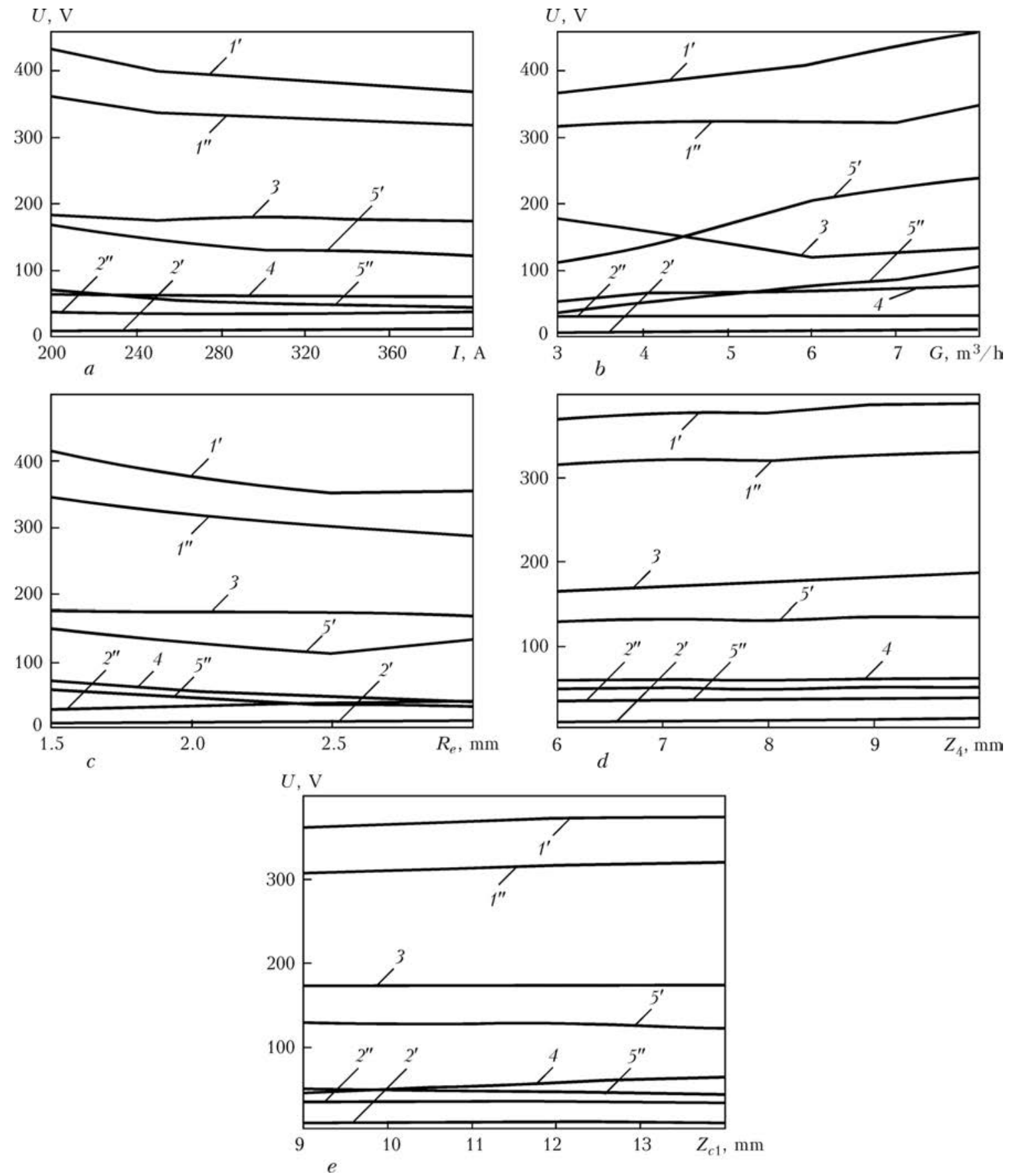

Figure 4. Dependencies of voltage drop across different parts of constricted arc column on current $(a)$, plasma gas flow rate $(b)$, radius $(c)$, length $(d)$ of plasmatron outlet nozzle and distance to cut item $(e): 1$ - total arc voltage drop; 2 - voltage drop inside hollow electrode; 3 - in plasma-forming channel; 4 - in arc open section; 5 - in cut cavity at plasmatron operation at reverse $\left(1^{\prime}, 5^{\prime}\right)$ and straight $\left(1^{\prime \prime}, 5^{\prime \prime}\right)$ polarity 

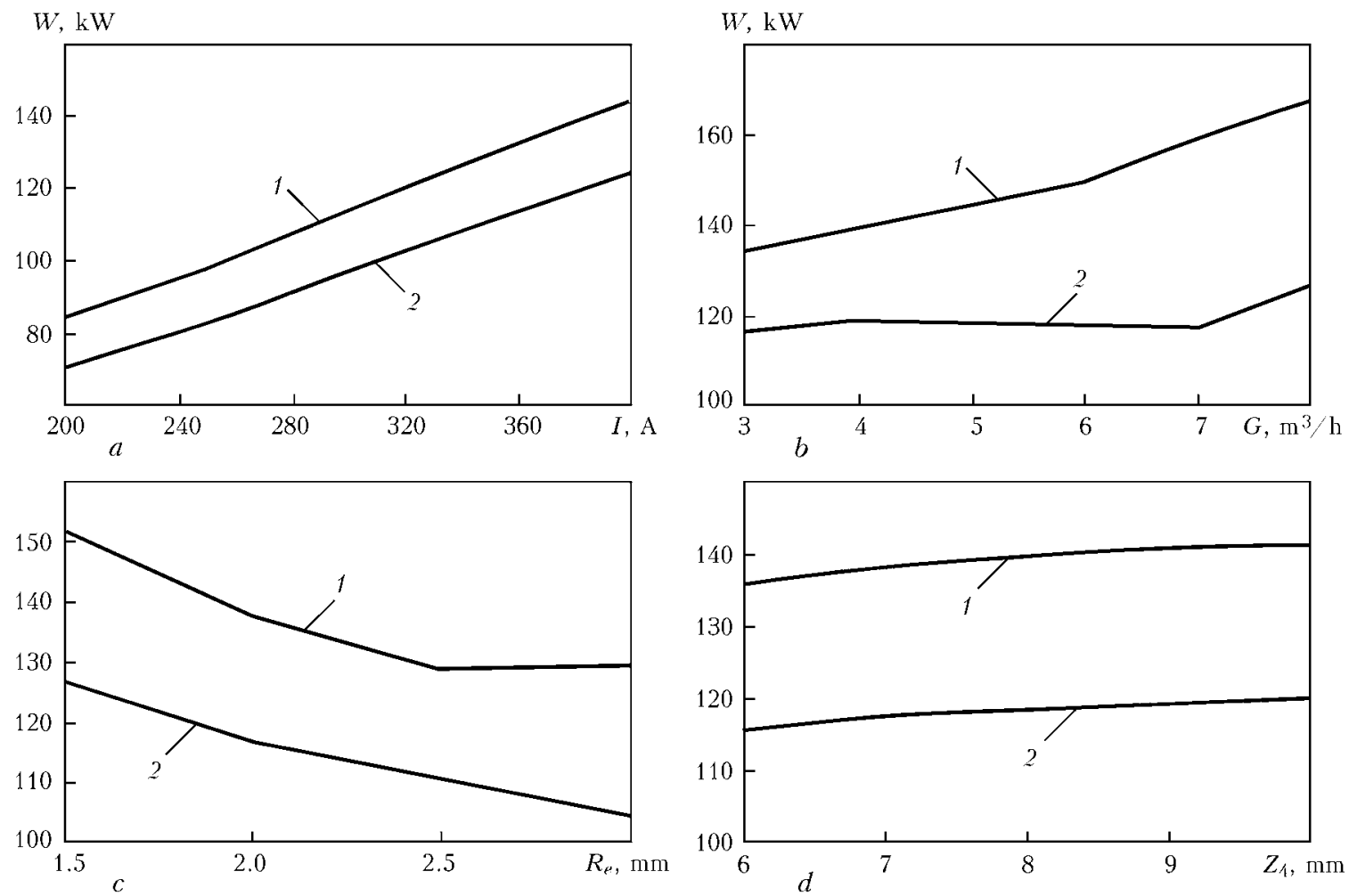

Figure 5. Dependencies of plasmatron total electric power on arc current $(a)$, plasma gas flow rate $(b)$, radius $(c)$ and length $(d)$ of plasmatron outlet nozzle at operation at reverse (1) and straight (2) polarity

entire depth of cut cavity that promotes increase of cutting process effectiveness.

Calculated total arc voltage, as well as voltage in arc individual regions, compared to experimentally measured voltage at cutting of $70 \mathrm{~mm}$ thick steel sheet in the considered plasmatron operation mode, are given in the Table.

Estimation of the value of total voltage drop across the cutting arc was performed under the following conditions: it was assumed that voltage drop near the regions of arc cathode and anode binding was equal to $10 \mathrm{~V}$ in sum; voltage drop in hollow electrode $\left(Z_{p}<z<Z_{1}\right)$ was evaluated using experimental findings, in keeping with which at SP cutting the arc binding is performed near the hollow electrode farther wall $\left(Z_{p}-Z_{1} \sim\right.$ $\sim 40 \mathrm{~mm}$ ), and at RP cutting - near the channel of plasma gas feeding $\left(Z_{p}-Z_{1} \sim 10 \mathrm{~mm}\right)$.

Let us now consider the results of calculation of integral electric and energy characteristics of the plasmatron at different sets of parameters of the process of straight and reverse polarity plasma-arc cutting (Figures 4 and 5).

As follows from Figure 4, $a$, calculated voltampere characteristic of the arc is a falling one in the considered plasmatron. Contrarily, dependence of arc voltage on plasma gas flow rate at constant $I_{\mathrm{a}}=315 \mathrm{~A}$ is a rising one. One can see from Figure 4, $b$ that at increase of plasma gas flow rate (above $5-6 \mathrm{~m}^{3} / \mathrm{h}$ ) restructuring of plasma flow in plasmatron channel occurs, that affects the change of flow parameters in the external region and in the cut area and, in its turn, leads to a change of the nature of voltage drop in these regions.

Influence of such geometrical parameters on arc voltage drop, as configuration of plasmatron working channel and distance to the item being cut, is given in Figure 4, $c-e$. It is shown that increase of arc column length leads also to increase of voltage drop value. Now, expansion of current channel, associated with increase of plasmatron outlet section diameter, leads to lowering of arc voltage.

Figure 5 gives the calculated dependencies of plasmatron electric power on its operation modes and geometrical parameters of working channel at plasmatron operation both at straight and at reverse polarity of the arc. Plasmatron electric power grows practically linearly at increase of arc current, as well as plasma gas flow rate (Figure $5, a, b)$. Drop of plasmatron power values occurs at increase of plasmatron outlet nozzle radius (Figure 5, c). This is related to increase of current channel dimensions and smaller voltage drop in the section, which corresponds to plasmatron working channel.

On the whole, derived results are indicative of the good prospects for application of the proposed approach to mathematical description of electric arc plasma, generated by cutting plasmatrons at their operation both at straight and 
at reverse polarity, as well as the possibility of application of the developed mathematical model and software for its computer realization, when retrofitting the design and selection of optimum operation modes of such plasmatrons.

\section{Conclusions}

1. A mathematical model of thermal, gas-dynamic and electromagnetic processes in arc plasma generated by cutting plasmatrons at their operation both at straight and reverse polarity was proposed. This model can be used for calculation of distributed and integral characteristics of arc plasma flow, generated by such plasmatrons inside the working channel, in open region of the arc, as well as in the cut cavity. The model allows conducting numerical analysis in broad ranges of variation of arc current and plasma gas flow rate, as well as geometrical parameters of plasmatron working channel and cut cavity.

2. Results of numerical studies of characteristics of arc plasma, generated by cutting plasmatron at reverse polarity, showed that these characteristics are significantly influenced by radius of plasmatron outlet nozzle, thickness of item being cut, as well as plasmatron operation parameters.

3. Thickness of item being cut has an essential influence on integral electric and energy characteristics of plasma arc. Therefore, cutting of thick items should be performed with application of power sources, providing operation in a broad range of working powers (up to $200 \mathrm{~kW}$ ) and voltages $(100-500 \mathrm{~V})$.

1. Shirshov, I.G. (1987) Plasma cutting. Leningrad: Mashinostroenie.

2. Kiselev, Yu.Ya. (2005) Investigation and development of technology and equipment for plasma-arc cutting of metals at reverse polarity: Syn. of Thesis for Dr. of Techn. Sci. Degree. Kishinev.

3. Shchitsyn, V.Yu. (2005) Improvement of plasmatron structures and technologies of plasma treatment of metals at reverse polarity: Syn. of Thesis for Cand. of Techn. Sci. Degree. Perm.

4. Engelsht, V.S., Asanov, D.S., Gurovich, V.Ts. et al. (1983) Mathematical modeling of electric arc. Frunze: Ilim.

5. Borisov, Yu.S., Chernyshov, A.V., Krivtsun, I.V. et al. (1994) Computer-aided simulation and experimental study of dusted plasma jets emitting into limited space. In: Proc. of Nat. Thermal Spray Conf. (Boston, USA, 1994), 361-366.

6. (1990) Theory of electric arc column. Ed. by M.F. Zhukov. Novosibirsk: Nauka.

7. Favalli, R.C., Szente, R.N. (1998) Physical and mathematical modeling of non transferred plasma torches. Brazilian J. Physics, 1(March), 25-34.

8. Kharlamov, M.Yu., Krivtsun, I.V., Korzhik, V.N. et al. (2007) Mathematical model of arc plasma generated by plasmatron with anode wire. The Paton Welding J., 12, 9-14.

9. Kharlamov, M.Yu., Krivtsun, I.V., Korzhik, V.N. et al. (2009) Refined mathematical model of the electric arc burning in plasmatron with external current-conducting wire. Ibid., 1, 42-45.

10. Lojtsyansky, L.G. (1973) Fluid and gas mechanics. Moscow: Nauka.

11. Boulos, M.I., Fauchais, P., Pfender, E. (1994) Thermal plasmas: Fundamentals and applications. Vol. 1. N.Y.; London: Plenum Press.

12. Launder, B.E., Spalding, D.B. (1990) The numerical computation of turbulent flows. Computer Methods in Appl. Mechanics and Eng., 8, 269-289.

13. Ievlev, V.M. (1975) Turbulent motion of high-temperature continua. Moscow: Nauka.

14. Wilcox, D.C. (1994) Turbulence modeling for CFD. La Canada:. DCW Ind. Inc.

15. Samarsky, A.A. (1971) Introduction to theory of difference schemes. Moscow: Nauka.

16. Anderson, D., Tannehill, J., Pletcher, R. (1990) Computational fluid mechanics and heat transfer. Vol. 1. Moscow: Mir.

17. Paskonov, V.M., Polezhaev, V.I., Chudov, L.A. (1984) Numerical modeling of processes of heat-, mass transfer. Moscow: Nauka.

Received 21.07.2015 